\title{
Research Article \\ Local Uniform Kadec-Klee Property (LUKK) and Modulus of (LUKK)
}

\author{
Yunan Cui $\mathbb{D}^{1}$ and Xiaoxia Wang $\mathbb{D}^{2}$ \\ ${ }^{1}$ Department of Mathematics, Harbin University of Science and Technology, Harbin 150080, China \\ ${ }^{2}$ Faculty of Mathematics and Computer Engineering, Ordos Institution of Applied Technology, Ordos 017000, China
}

Correspondence should be addressed to Xiaoxia Wang; syxyz2209@sina.com

Received 9 February 2020; Revised 19 May 2020; Accepted 22 June 2020; Published 7 July 2020

Academic Editor: Tomonari Suzuki

Copyright (c) 2020 Yunan Cui and Xiaoxia Wang. This is an open access article distributed under the Creative Commons Attribution License, which permits unrestricted use, distribution, and reproduction in any medium, provided the original work is properly cited.

\begin{abstract}
A new geometry property and two new moduli are introduced in Banach space. First, the concept of local uniform Kadec-Klee property $(L U K K)$ is introduced and the implication relationships between $(L U K K)$ and local near uniform convexity (LNUC), uniformly Kadec-Klee $(U K K),(H)$ are investigated in Banach space. Furthermore, the modulus $P_{X}^{L}(\varepsilon)$ of $(L U K K)$ and the modulus $\Delta_{X}^{L}(\varepsilon)$ of $(L N U C)$ are introduced and the relationship of size between $P_{X}^{L}(\varepsilon)$ and $\Delta_{X}^{L}(\varepsilon)$ is also investigated in Banach space. Finally, several formulas for $P_{X}^{L}(\varepsilon)$ are calculated in classical Banach space $l^{P}$.
\end{abstract}

\section{Introduction}

Let $(X,\|\cdot\|)$ be a Banach space, $X^{*}$ be the dual space of $X$. By $U(X)$ and $S(X)$, we denote the closed unit ball and the unit sphere of Banach space $X$, respectively. By $c o(A)$ and $\overline{c o}(A)$, we denote the convex hull and closed convex hull of the set $A$, respectively. $x_{n} \longrightarrow^{w} x$ as $n \longrightarrow \infty$ denotes $\left\{x_{n}\right\}$ is weakly converges to $x$ as $n \longrightarrow \infty$.

It is well known that the condition equivalent to near uniform convexity $(N U C)$ was independently formulated in [1] (see also [2]). Recall that a notion of noncompactness with Hausdorff measure and Kuratowski measure of a set (see [3]). Let $A$ be a bounded subset of $X$. Fix $\varepsilon \in[0,1]$ for all convex closed sets $A \subset U(X)$ with $\mu(A) \geq \varepsilon$, we put

$$
\begin{aligned}
& \Delta_{X}(\varepsilon)=\inf \{1-d(\theta, A): A \subset U(X), A=c o(A), \mu(A) \geq \varepsilon\}, \\
& \widehat{\Delta}_{X}(\varepsilon)=\inf \{1-d(\theta, A): A \subset U(X), A=c o(A), \alpha(A) \geq \varepsilon\},
\end{aligned}
$$

where

$$
\begin{aligned}
\mu(A)= & \inf \{\varepsilon>0: \text { A can be covered with a finite number of } \\
& \text { sets of radii smaller than } \varepsilon\}, \\
\alpha(A)= & \inf \{\varepsilon>0: \text { A can be covered by finitely many sets } \\
& \text { with diameter }<\varepsilon\} .
\end{aligned}
$$

The functions $\Delta_{X}(\varepsilon)$ and $\widehat{\Delta}_{X}(\varepsilon)$ are called the moduli of noncompact convexity with Hausdorff measure and Kuratowski measure of $X$, respectively. It is clear that $X$ is $(N U C)$ if and only if $\Delta_{X}(\varepsilon)>0$ for every $\varepsilon \in(0,1]$. Other properties of $\Delta_{X}(\varepsilon)$ were investigated in [4-7].

Recall also that a function

$$
\varepsilon_{1}(X)=\sup \left\{\varepsilon: \Delta_{X}(\varepsilon)=0\right\} .
$$


J. Banas' proved that if $\varepsilon_{1}(X)<1$, then $X$ is reflexive and has normal structure (see [3]).

The modulus of $(U K K)$ was introduced in [8] by J. R. Partington that is $P_{X}:[0,1] \longrightarrow[0,1]$

$$
\begin{aligned}
P_{X}(\varepsilon)= & \inf \{1-\|x\|: \exists x \in U(X) \text { and } \\
& \left.\left\{x_{n}\right\} \subset U(X) \text { s.t. } x_{n} \stackrel{w}{\longrightarrow} x \text { and } \operatorname{sep}\left(x_{n}\right) \geq \varepsilon\right\},
\end{aligned}
$$

where $\operatorname{sep}\left(x_{n}\right)=\inf \left\{\left\|x_{n}-x_{m}\right\|: m \neq n\right\}$. He proved that $X$ has $(U K K)$ property if and only if $P_{X}(\varepsilon)>0$ whenever $\varepsilon \in[0,1]$. He also proved that the function $P_{X}$ is nondecreasing on $[0,1]$.

There are many recent papers concerning the Kadec-Klee property, such as Kadec.CKlee property and fixed points and Dual Kadec-Klee property and fixed points studied by Jean Saint Raymond (see $[9,10]$ ). In a recent paper [11], Maciej Ciesielski, Paweł Kolwicz, and Ryszard Płuciennik were interested in local approach to Kadec"CKlee property in symmetric function spaces. Moreover, normal structure and moduli of $(U K K),(N U C)$, and $(U K K)^{*}$ in Banach spaces have been deeply investigated by Satit Saejung and Ji Gao. The new kind of Banach spaces: (semi-UKK), (semi-NUC), modulus of (semi-UKK), and modulus of $($ semi-NUC) are introduced in terms of this $\mathrm{u}$-separation measure in their paper.(see [12]).

Since Banach space is more extensive than Hilbert space, it is quite difficult to describe its geometry structure. An effective method is to introduce new geometric properties for Banach space and to define an appropriate function, usually called a modulus or a geometric constant. Because the range of values of these geometric constants directly determines the existence of some geometric properties; therefore, many scholars are interested to calculate the modules and constants of some specific spaces. The starting point of the present paper is the observation that the $(\mathrm{U} K K)$ property can be localized. We call this new property named the local uniform Kadec-Klee property $(L U K K)$, and we observe that it lies strictly between $(H)$ and $(U K K)$ properties. By using the same localized method, we localize the modulus $\left(P_{X}(\varepsilon)\right)$ of $(U K K)$ introduced by J. R. Partington and the modulus of noncompact convexity with Hausdorff measure $\left(\Delta_{X}(\varepsilon)\right)$ obtain two new moduli $P_{X}^{L}(\varepsilon)$ and $\Delta_{X}^{L}(\varepsilon)$, and we observe that $\Delta_{X}^{L}(\varepsilon) \geq P_{X}^{L}(\varepsilon)$.

\section{Preliminaries}

Before starting with our results, we need to recall some notions and a lemma in [13-15].

We say that a Banach space $X$ has $(H)$ property if for any $x \in S(X),\left\{x_{n}\right\} \subset X, \lim _{n \longrightarrow \infty}\left\|x_{n}\right\|=\|x\|$ and $x_{n} \longrightarrow^{w} x$, then $\lim _{n \rightarrow \infty}\left\|x_{n}-x\right\|=0$.

We say that a Banach space $X$ has Kadec-Klee (UKK) property if for every $\varepsilon>0$ there exists $0<\delta<1$ such that $\left\{x_{n}\right\} \subset U(X), \quad x_{n} \longrightarrow^{w} x$ and $\operatorname{sep}\left(x_{n}\right) \geq \varepsilon$ then $x \in B_{\delta}(0)$ where $B_{\delta}(0)=\{x:\|x\| \leq \delta\}$.

We say that a Banach space $X$ has near uniform convexity property (NUC) if for every $\varepsilon>0$ there exists $0<\delta=\delta(\varepsilon)<1$ such that $\left\{x_{n}\right\} \subset U(X)$ with $\operatorname{sep}\left(x_{n}\right) \geq \varepsilon$ then there is a $N \geq 1$ and scalars $\lambda_{1}, \cdots, \lambda_{N} \geq 0$ with $\sum \lambda_{n}=1$ such that $\left\|\sum \lambda_{n} x_{n}\right\| \leq$ $1-\delta$.

We say that a Banach space $X$ has local near uniform convexity property $(L N U C)$ if for every $\varepsilon>0$ and $x \in U(X)$ there exists $\delta=\delta(x, \varepsilon)>0$ such that for any sequence $\left\{x_{n}\right\} \subset$ $U(X)$ with $\operatorname{sep}\left(x_{n}\right) \geq \varepsilon$ then

$$
\operatorname{co}\left(\left\{x_{n}, x\right\}\right) \cap B_{\delta}(0) \neq \varnothing .
$$

i.e.,

$$
\left\|\lambda_{0} y+\left(1-\lambda_{0}\right) x\right\|<\delta \text { where } y \in \operatorname{co}\left(\left\{x_{n}\right\}\right)
$$

for some $\lambda_{0} \in(0,1)$ and $y \in \operatorname{co}\left(x_{n}\right)$ (see [16]).

Lemma 1. Let $X$ be a Banach Space, $\left\{x_{n}\right\} \subset X$ and $x_{n} \longrightarrow^{w} x_{0}$. Then,

$$
\left\{x_{0}\right\}=\cap_{n=1}^{\infty} \overline{c o}\left(\left\{x_{k}\right\}_{k \geq n}\right) .
$$

\section{Materials and Methods}

In this paper, we take Kutazrova and Bor-Luh Lin's approach to localize the $(U K K)$ property and obtain the $(L U K K)$ property. By using the same localized method, we localize the modulus $\left(P_{X}(\varepsilon)\right)$ of $(U K K)$ which introduced by Partington and the modulus of noncompact convexity with Hausdorff measure $\left(\Delta_{X}(\varepsilon)\right)$ and obtain two new moduli $P_{X}^{L}(\varepsilon)$ and $\Delta_{X}^{L}(\varepsilon)$; then, we study the relationship of size between $P_{X}^{L}(\varepsilon)$ and $\Delta_{X}^{L}(\varepsilon)$ in Banach space by using the Corollary of Hahn-Banach Theorem and the weak lower semi continuity of norm.

\section{Results and Discussion}

We begin this section by formulating some definitions.

Definition 2. A Banach space $X$ is said to have local uniform Kadec - Klee property (LUKK), for every $\varepsilon>0$ and $x \in S(X)$ there exists $\delta>0$ such that if $\left\|x_{n}\right\| \leq 1, x_{n} \longrightarrow^{w} x_{0}$, $\operatorname{sep}\left(x_{n}\right) \geq \varepsilon$ and $\left\|x_{n}-x\right\| \geq \varepsilon$ then

$$
\operatorname{co}\left(x_{0}, x\right) \cap B_{\delta}(0) \neq \varnothing \text { where } B_{\delta}(0)=\{x:\|x\| \leq \delta\},
$$

i.e.,

$$
\left\|\lambda_{0} x_{0}+\left(1-\lambda_{0}\right) x\right\|<\delta
$$

for some $\lambda_{0} \in(0,1)$.

Definition 3. Let $X$ be a Banach Space. For every $\varepsilon>0$ and $x \in S(X)$

$$
\begin{aligned}
P_{X}^{L}(\varepsilon)= & \inf \left\{1-\left\|\operatorname{co}\left(x, x_{0}\right)\right\|: \mid x_{n} \| \leq 1, x_{n} \stackrel{w}{\longrightarrow} x_{0},\right. \\
& \left.\operatorname{sep}\left(x_{n}\right) \geq \varepsilon,\left\|x_{n}-x\right\| \geq \varepsilon\right\},
\end{aligned}
$$


is said to be the modulus of $(L U K K)$ property or local Partington's coefficient.

Definition 4. For every $\varepsilon>0$ and $x \in S(X)$, we put

$$
\begin{gathered}
\Delta_{X}^{L}(\varepsilon)=\inf \left\{1-d\left(\theta, \operatorname{co}\left(x, \operatorname{co}\left(x_{n}\right)\right)\right):\left\|x_{n}\right\| \leq 1,\right. \\
\left.\alpha\left(\operatorname{co}\left(x, \operatorname{co}\left(x_{n}\right)\right)\right) \geq \varepsilon,\left\|x_{n}-x\right\| \geq \varepsilon\right\},
\end{gathered}
$$

where

$$
\begin{aligned}
\alpha(A)= & \inf \{\varepsilon>0: \text { A can be covered by finitely many sets } \\
& \text { with diameter }<\varepsilon\}
\end{aligned}
$$

$\Delta_{X}^{L}(\varepsilon)$ is said to be the modulus of (LNUC) with Kuratowski measure.

Corollary 5. If a Banach space X has (LUKK) property, then $X$ has $(H)$ property.

Proof. We prove the contrapositive. Suppose $X$ does not have $(H)$ property, then there exists $\left\{x_{n}\right\} \subset S(X)$ and $x_{0} \in S(X)$ such that although $x_{n} \longrightarrow^{w} x_{0}$ as $n \longrightarrow \infty$, we still have $x_{n}{ }^{1 / 2} x_{0}$ what means there exists $\varepsilon_{0}>0$ and $n_{k}>n$ for any $n \in N$ such that $\left\|x_{n_{k}}-x\right\| \geq \varepsilon_{0}$ this implies that $\operatorname{sep}\left(x_{n_{k}}\right) \geq \varepsilon_{0}$ holds.

$X$ has $(L U K K)$ property, for $\varepsilon_{0}>0$ and $x_{0} \in S(X)$ mentioned above, there exists $\delta \in(0,1)$ such that

$$
\operatorname{co}\left(x_{0}, x_{0}\right) \cap B_{\delta}(0) \neq \varnothing,
$$

i.e.,

$$
x_{0} \cap B_{\delta}(0) \neq \varnothing,
$$

this shows $\left\|x_{0}\right\| \leq \delta<1$, a contradiction. Thus, the assumption does not hold.

The following conclusion follows from the definitions of $(L U K K)$ and $(U K K)$.

Corollary 6. If Banach space $X$ has $(U K K)$ property, then $X$ has (LUKK) property.

It follows from previous Corollaries, we conclude the following Corollary.

Corollary 7. For every Banach space $X$, the implication $(U K K) \Longrightarrow(L U K K) \Longrightarrow(H)$ holds. paper.

We are now ready to prove the main theorems of this

Theorem 8. If a Banach space $X$ is (LNUC), then $X$ has (LUKK) property.
Proof. Suppose that $X$ does not have $(L U K K)$ property. Then, there exists $\varepsilon_{0}>0, x_{0} \in S(X)$ and $\left\{y_{n}\right\} \subset U(X)$ with se $p\left(y_{n}\right) \geq 2 \varepsilon_{0}, y_{n} \longrightarrow^{w} y_{0}$ with

$$
\operatorname{co}\left(\left\{x_{0}, y_{0}\right\}\right) \cap B_{1-1 / n}(0)=\varnothing
$$

Since $X$ has $(L N U C)$ property, for $\varepsilon_{0}>0$ and $x_{0} \in S(X)$ mentioned above, there exists $\delta=\delta\left(x_{0}, \varepsilon_{0}\right)>0$ such that $\operatorname{co}\left(x_{0}, \operatorname{co}\left(\left\{y_{n}\right)\right\}\right) \cap B_{1-\delta}(0) \neq \varnothing$, which means for some $\lambda_{0} \in$ $[0,1]$ and $y^{\prime} \in \operatorname{co}\left(\left\{y_{n}\right\}\right)$, we have

$$
\left\|\lambda_{0} x_{0}+\left(1-\lambda_{0}\right) y^{\prime}\right\| \leq 1-\delta
$$

from (15) it follows that for any $\lambda \in[0,1]$ we have

$$
\left\|\lambda x_{0}+(1-\lambda) y_{0}\right\|>1-\frac{1}{n} \longrightarrow 1, n \longrightarrow \infty
$$

Case (i) if $y_{0}=y^{\prime}$, then (16) contradicts with (17).

Case (ii) if $y_{0} \neq y^{\prime}$, since $y_{n} \longrightarrow^{w} y_{0}$, then by Lemma 1 , we have $\left\{y_{0}\right\}=\cap_{n=1}^{\infty} \overline{c o}\left(\left\{y_{k}\right\}_{k \geq n}\right)$, since $y^{\prime} \in \operatorname{co}\left(\left\{y_{n}\right\}\right)$ then for any $x \in S(X)$ and $\lambda \in[0,1]$. Let

$$
\begin{aligned}
Y_{x}(\delta) & =\{y \in B(X): 1-\delta<\|\lambda x+(1-\lambda) y\|<1\} \\
\psi(\lambda) & =\|\lambda x+(1-\lambda) y\|, \\
L & =\{\lambda \in[0,1]: \psi(\lambda) \leq 1-\delta\} \\
\lambda^{\prime} & =\inf \{\lambda \in[0,1]: \psi(\lambda) \leq 1-\delta\},
\end{aligned}
$$

It is obvious that $\psi(0) \leq \psi(1)=1, L \neq \varnothing$, and $\psi\left(\lambda^{\prime}\right)=$ $1-\delta$. From (16), we get

$$
y^{\prime} \in Y_{x_{0}}(\delta)
$$

For another facts, for $\lambda \in[0,1]$, let $z_{0}=\lambda^{\prime} y^{\prime}+\left(1-\lambda^{\prime}\right) x_{0}$; then, we have

$$
\begin{aligned}
\left\|\lambda z_{0}+(1-\lambda) x_{0}\right\| & =\left\|\lambda \lambda^{\prime} y^{\prime}+\left(1-\lambda \lambda^{\prime}\right) x_{0}\right\| \\
& =\psi\left(\lambda \lambda^{\prime}\right)>1-\delta
\end{aligned}
$$

i.e.,

$$
\left\|\lambda \lambda^{\prime} y^{\prime}+\left(1-\lambda \lambda^{\prime}\right) x_{0}\right\|>1-\delta
$$

Thus, $y^{\prime} \in Y_{x_{0}}(\delta)$ which contradicts with (19); therefore, the assumption is not true.

Theorem 9. For every Banach space $X$, we have $P_{X}^{L}(\varepsilon) \geq \Delta_{X}^{L}(\varepsilon)$. 
Proof. Fix $\varepsilon \in[0,1]$ and take an arbitrary sequence $\left\{x_{n}\right\} \subset$ $U(X)$ with $\operatorname{sep}\left(x_{n}\right) \geq \varepsilon, x_{n} \longrightarrow^{w} x_{0}$ as $n \longrightarrow \infty, \alpha\left(x_{n}\right) \geq \varepsilon$. For every $x \in S(X)$ and some $\lambda \in(0,1)$, we let

$$
\begin{aligned}
& y_{n}=\lambda x+(1-\lambda) x_{n}, \\
& y_{0}=\lambda x+(1-\lambda) x_{0} .
\end{aligned}
$$

By the corollary of Hahn-Banach theorem, there exists $f_{0} \in S\left(X^{*}\right)$ such that $f_{0}\left(y_{n}\right)=\left\|y_{n}\right\|$. Picking $\eta>0$ be small enough and considering the following set

$$
D\left(f_{0}, \eta\right)=\left\{y \in U(X): f_{0}(y) \geq\left\|y_{0}\right\|-\eta\right\} .
$$

It is obvious that the set $D\left(f_{0}, \eta\right)$ is closed, convex, and

$$
d\left(\theta, D\left(f_{0}, \eta\right)\right) \geq\left\|y_{0}\right\|-\eta .
$$

Since $x_{n} \longrightarrow^{w} x_{0}$, then

$$
f_{0}\left(y_{n}\right) \longrightarrow f_{0}\left(y_{0}\right)=\left\|y_{0}\right\| \text {. }
$$

Then, there exists $n_{0} \in N$ such that

$$
f_{0}\left(y_{n}\right) \geq\left\|y_{0}\right\|-\eta \text { when } n \geq n_{0},
$$

this implies that the set $Y_{n_{0}}=\left\{y_{n}: n \geq n_{0}\right\}$ is a subset of $D\left(f_{0}, \eta\right)$. Then, we get

$$
d\left(\theta, \operatorname{co}\left(Y_{n_{0}}\right) \geq\left\|y_{0}\right\|-\eta\right.
$$

Since $\alpha\left(Y_{n_{0}}\right) \geq \varepsilon$, then

$$
\alpha\left(\operatorname{co}\left(Y_{n_{0}}\right)\right) \geq \varepsilon \text { and } \Delta_{X}^{L}(\varepsilon) \leq 1-d\left(\theta, \operatorname{co}\left(Y_{n_{0}}\right)\right) .
$$

from (27) it follows that

$$
1-d\left(\theta, \operatorname{co}\left(Y_{n_{0}}\right)\right) \leq 1-\left\|y_{0}\right\|+\eta .
$$

Consequently,

$$
\begin{aligned}
\Delta_{X}^{L}(\varepsilon) & \leq 1-d\left(\theta, c o\left(Y_{n_{0}}\right)\right) \leq 1-\left\|y_{0}\right\|+\eta, \\
1-\left\|y_{0}\right\| & \geq \Delta_{X}^{L}(\varepsilon)-\eta .
\end{aligned}
$$

Thus, $P_{X}^{L}(\varepsilon) \geq \Delta_{X}^{L}(\varepsilon)-\eta$. Since $\eta>0$ is small enough, then we get $P_{X}^{L}(\varepsilon) \geq \Delta_{X}^{L}(\varepsilon)$ and the proof is complete.

Theorem 10. For Banach space $\mathbb{P}^{p}(1<p<\infty)$, we have $P_{p^{p}}^{L}(\varepsilon) \geq 1-\left(1-(\varepsilon / 2)^{p}\right)^{1 / p}$.

Proof. For every $\varepsilon>0, x \in S\left(p^{p}\right)$ and $\lambda \in(0,1)$, let $\left\{x_{n}\right\} \subset$ $U\left(p^{P}\right)$ such that $x_{n} \longrightarrow^{w} x_{0}$, sep $\left(x_{n}\right) \geq \varepsilon$ and $\left\|x_{n}-x\right\| \geq \varepsilon$. Let

$$
\begin{aligned}
& y_{n}=\lambda x+(1-\lambda) x_{n}, \\
& y_{0}=\lambda x+(1-\lambda) x_{0},
\end{aligned}
$$

then $y_{n} \longrightarrow^{w} y_{0}$. By the weak lower semicontinuity of norm function, we get

$$
\left\|y_{0}\right\| \leq \inf \lim _{n}\left\|y_{n}\right\| .
$$

Then, there exists a subsequence $\left\{y_{n_{k}}\right\} \subseteq\left\{y_{n}\right\}$ and $K \in N$ such that $\left\|y_{0}\right\| \leq\left\|y_{n_{k}}\right\|$ for all $k>K$. Hence,

$$
\begin{aligned}
\left\|y_{0}\right\|^{p} & \leq \min \left\{\left\|y_{n_{i}}\right\|^{p},\left\|y_{n_{j}}\right\|^{p}: i, j>k\right\} \\
& =\frac{\left|\left\|y_{n_{i}}\right\|^{p}+\left\|y_{n_{j}}\right\|^{p}\right|-\left|\left\|y_{n_{i}}\right\|^{p}-\left\|y_{n_{j}}\right\|^{p}\right|}{2} \\
& \leq \frac{2-\left\|y_{n_{i}}-y_{n_{j}}\right\|^{p}}{2} .
\end{aligned}
$$

Since

$$
\left\|y_{n_{i}}-y_{n_{j}}\right\|=\lambda\left\|x_{n_{i}}-x_{n_{j}}\right\| \geq \lambda \varepsilon,
$$

then we get

$$
\begin{aligned}
\left\|y_{0}\right\|^{p} & \leq 1-\frac{\left\|y_{n_{i}}-y_{n_{j}}\right\|^{p}}{2} \leq 1-\frac{(\lambda \varepsilon)^{p}}{2}, \\
\left\|y_{0}\right\| & \leq\left(1-\frac{(\lambda \varepsilon)^{p}}{2}\right)^{1 / p}, \\
1-\left\|y_{0}\right\| & \geq 1-\left(1-\frac{\lambda^{p} \varepsilon^{p}}{2}\right)^{1 / p},
\end{aligned}
$$

thus

$$
P_{p^{p}}^{L}(\varepsilon) \geq 1-\left(1-\frac{\lambda^{p} \varepsilon^{p}}{2}\right)^{1 / p} \text { where } 1<p<\infty,
$$

it follows that

$$
P_{l^{p}}^{L}(\varepsilon) \geq 1-\left(1-\frac{\varepsilon^{p}}{2}\right)^{1 / p} \text { where } 1<p<\infty .
$$

Theorem 11. If $X$ is a reflexive Banach space, then for any $\varepsilon \in[0,2]$, we have $\Delta_{X}^{L}(\varepsilon) \geq P_{X}^{L}(\varepsilon / 2)$.

Proof. Fix $\varepsilon \in[0,2]$. Take $\left\{x_{n}\right\} \subset U(X)$ with $\alpha\left(x_{n}\right) \geq \varepsilon$ then $\alpha\left(\operatorname{co}\left(x_{n}\right)\right) \geq \varepsilon$; here, we let

$$
y_{n}=\lambda x+(1-\lambda) x_{n},
$$

for every $x \in S(X)$ and $\lambda \in[0,1]$. Thus, there exists $\left\{z_{n}\right\} \subset$ $\operatorname{co}\left(y_{n}\right)$ such that $\operatorname{sep}\left(z_{n}\right) \geq \varepsilon / 2$. By the reflexivity of $X$, there exists subsequence $\left\{z_{n_{k}}\right\} \subset\left\{z_{n}\right\} \subset \operatorname{co}\left(y_{n}\right)$ and $z_{0} \in U(X)$ such that $z_{n_{k}} \longrightarrow^{w} z_{0}$. It is obvious that

$$
\begin{aligned}
& \left\|\operatorname{co}\left(z_{0}, x\right)\right\| \leq 1-P_{X}^{L}\left(\frac{\varepsilon}{2}\right) \\
& 1-P_{X}^{L}\left(\frac{\varepsilon}{2}\right) \geq \sup \left\{\left\|\alpha z_{0}+(1-\alpha) x\right\|: \alpha \in(0,1)\right\} .
\end{aligned}
$$


And consequently,

$$
\left\|z_{0}\right\| \leq 1-P_{X}^{L}\left(\frac{\varepsilon}{2}\right) \text { and } z_{0} \in \operatorname{co}\left(z_{n_{k}}\right),
$$

this implies that

$$
\begin{aligned}
d\left(\theta, \operatorname{co}\left(\operatorname{co}\left(x, x_{n}\right)\right)\right) & =d\left(\theta, \operatorname{co}\left(y_{n}\right)\right) \leq d\left(\theta, \operatorname{co}\left(z_{n}\right)\right) \\
& \leq d\left(\theta, \operatorname{co}\left(z_{n_{k}}\right)\right) \leq\left\|z_{0}\right\| \leq 1-P_{X}^{L}\left(\frac{\varepsilon}{2}\right) .
\end{aligned}
$$

From (41), it follows that

$$
1-d\left(\theta, \operatorname{co}\left(\operatorname{co}\left(x, x_{n}\right)\right)\right) \geq P_{X}^{L}\left(\frac{\varepsilon}{2}\right) .
$$

Thus, $\Delta_{X}^{L}(\varepsilon) \geq P_{X}^{L}(\varepsilon / 2)$ for any $\varepsilon \in[0,2]$, and the proof is complete.

\section{Conclusions}

In this paper, we introduce a new geometric property ( $L U K K)$ that lies between two classical geometric properties (UKK) and $(H)$. Moreover, two new moduli $P_{X}^{\mathrm{L}}(\varepsilon)$ and $\Delta_{X}^{L}(\varepsilon)$ for $(L U K K)$ and $(L N U C)$ are introduced in Banach spaces; these new notions introduced in our paper play a very significant role in some recent trends of the geometric theory of Banach spaces. Furthermore, we give some further facts concerning the implication between (LUKK) and (LNUC). Moreover, the relationship of size between the moduli $P_{X}^{L}(\varepsilon)$ and $\Delta_{X}^{L}(\varepsilon)$ is discussed in Banach spaces, and $P_{X}^{L}(\varepsilon)$ is calculated in classical Banach spaces $l_{p}$ meanwhile. We believe that these introduced concepts will be useful and can be used to further solve the problems of accurately reflecting the shape and geometric structure of the unit sphere in Banach space.

\section{Data Availability}

No data were used to support this study.

\section{Conflicts of Interest}

The authors declare that there is no conflict of interest regarding the publication of this paper.

\section{Acknowledgments}

The authors are grateful to the referee for comments which improved the paper. This paper is supported by "The National Science Foundation of China" (11871181); "The Science Research Project of Inner Mongolia Autonomous Region" (NJZY18253); "The Science Research Project of Ordos Institution of Applied Technology" (KYYB2017014).

\section{References}

[1] K. Goebel and T. Sekowski, "The modulus of noncompact convexity," Annales Universitatis Mariae Curie-Sklodowska, sectio $A$ A, vol. 38, pp. 41-48, 1984.
[2] K. Goebel and W. A. Kirk, Topics in metric fixed point theory, Cambridge University Press, Cambridge, 1990.

[3] J. Banas and K. Goebel, Measures of noncompactness in Banach spaces, Lecture Notes in Pure and Application Mathematical, Marcel Dekker, New York, 1980.

[4] J. Banas, "On modulus of noncompact convexity and its properties," Canadian Mathematical Bulletin, vol. 30, no. 2, pp. 186-192, 1987.

[5] J. Banas, "Compactness conditions in the geometric theory of Banach spaces," Nonlinear Analysis, vol. 16, no. 7-8, pp. 669682, 1991.

[6] S. Prus, "Banach spaces with the uniform Opial property," Nonlinear Analysis, vol. 18, no. 8, pp. 697-704, 1992.

[7] S. Prus, "On the modulus of noncompact convexity of a Banach space," Archiv der Mathematik, vol. 63, no. 5, pp. 441-448, 1994.

[8] J. R. Partington, "On nearly uniformly convex Banach spaces," Mathematical Proceedings of the Cambridge Philosophical Society, vol. 93, no. 1, pp. 127-129, 1983.

[9] J. Saint Raymond, "Kadec-Klee property and fixed points," Journal of Functional Analysis, vol. 266, no. 8, pp. 54295438, 2014.

[10] J. Saint Raymond, "Dual Kadec-Klee property and fixed points," Journal of Functional Analysis, vol. 272, no. 9, pp. 3825-3844, 2017.

[11] M. Ciesielski, P. Kolwicz, and R. Płuciennik, "Local approach to Kadec-Klee properties in symmetric function spaces," Journal of Mathematical Analysis and Applications, vol. 426, no. 2, pp. 700-726, 2015.

[12] S. Saejung and J. Gao, "Normal structure and moduli of UKK, NUC, and UKK* in Banach spaces," Applied Mathematics Letters, vol. 25, no. 10, pp. 1548-1553, 2012.

[13] R. Huff, "Banach spaces which are nearly uniformly convex," Rocky Mountain Journal of Mathematics, vol. 10, no. 4, pp. 743-750, 1980.

[14] B.-L. Lin and W. Y. Zhang, "Some geometric properties related to uniformly convexity of Banach spaces, function spaces," Lecture Notes in Pure and Applied Mathematics, vol. 136, pp. 281-291, 1992.

[15] S. Rolewicz, "On $\Delta$-uniform convexity and drop property," Studia Mathematica, vol. 87, no. 2, pp. 181-191, 1987.

[16] D. N. Kutzarova and B.-L. Lin, "Locally k-nearly uniformly convex Banach spaces," Mathematica Balkanica, vol. 8, pp. 203-210, 1994. 